\title{
Water-Soluble Chitosan Nanoparticles Inhibit Hypercholesterolemia Induced by Feeding a High-Fat Diet in Male Sprague-Dawley Rats
}

\author{
Yi Tao, Hongliang Zhang, Bing Gao, Jiao Guo, Yinming Hu, and Zhengquan Su \\ Key Unit of Modulating Liver to Treat Hyperlipemia SATCM and Lipid Metabolism Laboratory of 3rd Level SATCM, \\ Guangdong Pharmaceutical University, Guangzhou 510006, China \\ Correspondence should be addressed to Zhengquan Su, suzhq@scnu.edu.cn
}

Received 27 May 2010; Revised 7 July 2010; Accepted 10 July 2010

Academic Editor: Libo Wu

Copyright () 2011 Yi Tao et al. This is an open access article distributed under the Creative Commons Attribution License, which permits unrestricted use, distribution, and reproduction in any medium, provided the original work is properly cited.

Chitosan, a deacetylated product of chitin, has been demonstrated to lower cholesterol in humans and animals. However, chitosan is not fully soluble in water which would influence absorption in the human intestine. In addition, water-soluble chitosan (WSC) has higher reactivity compared to chitosan. The present study was designed to clarify the effects of WSC and water-soluble chitosan nanoparticles (WSC-NPs) on hypercholesterolemia induced by feeding a high-fat diet in male Sprague-Dawley rats. WSC-NPs were prepared by the ionic gelation method and the spray-drying technique. The nanoparticles were spherical in shape and had a smooth surface. The mean size of WSC-NPs was $650 \mathrm{~nm}$ variing from 500 to $800 \mathrm{~nm}$. Results showed that WSC-NPs reduced the blood lipids and plasma viscosity significantly and increased the serum superoxide dismutase (SOD) activities significantly. This paper is the first report of the lipid-lowering effects of WSC-NPs suggesting that the WSC-NPs could be used for the treatment of hypercholesterolemia.

\section{Introduction}

Dyslipidemia, including hypercholesterolemia, hypertriglyceridemia, or their combination, is a major risk factor for cardiovascular disease. Generally, dyslipidemia is characterized by increased fasting concentrations of total cholesterol (TC), triglycerides (TG), and low-density lipoprotein cholesterol (LDL-C), in conjunction with decreased concentrations of high-density lipoprotein cholesterol (HDL-C) [1]. At present, these lipid imbalances are most routinely treated with pharmacological therapy. However, many cholesterollowering agents, especially statins, are associated with severe side effects [2]. In light of this, there has been great interest in the influence of dietary fibers, such as chitosan, on cholesterol absorption in the intestine.

Chitosan is a natural cationic polysaccharide consisting of (1-4)-2-amino-2-deoxy-D-glucopyranosyl units. It breaks down slowing to harmless products (amino sugars), which are completely absorbed by the human body [3]. Due to its nontoxicity and high biocompatibility, chitosan has been formulated as dietary supplements, as carrier for oral peptide and protein drug delivery, as targeted drug delivery, and in the pharmaceutical and biomedical fields [4-6]. Due to the existence of amino groups, chitosan possesses positive charge, so it can bind negatively charged substrates such as lipids and bile acids. Chitosan also interfere with emulsification of neutral lipids by binding them with hydrophobic bonds [7]. Several studies have shown that chitosan has cholesterol-lowering properties both in animals and humans $[8,9]$.

However, chitosan has high viscosity and is not fully soluble in water, but it is in acidic solutions. Such properties of chitosan would decrease its absorption in the human intestine because most animal intestines, especially the human gastrointestinal tract, do not possess enzymes such as chitinase and chitosanase [10]. WSC has lower viscosity and is soluble in water. Subsequently, it seems to be readily absorbed in vivo. And, WSC has been reported to have the health benefits such as immunity regulation, antitumor, liver protection, blood lipids lowering, and antidiabetic 
and antioxidant properties $[11,12]$. In particular, previous studies revealed that the WSC was effective at lipid lowering compared to chitosan [13].

Furthermore, nanoparticles show some specific characteristics such as an increase of stability of therapeutic agents, controlled- and sustained-release properties, and the deeply penetration into tissues through fine capillaries [14]. We have prepared the WSC-NPs as a carrier to load the protein drug by the ionic gelation method [15]. And, WSC-NPs have the better solubility for the big total surface area and lower viscosity than WSC. Therefore, this study examined the effects of WSC and WSC-NPs on hypercholesterolemia induced by feeding a high-fat diet in rats.

\section{Materials and Methods}

2.1. Chemicals. WSC was purchased from Shandong Aokang Biotech Ltd (Shandong, China). The viscosity was more than $200 \mathrm{cps}$, and deacetylation value was $85 \%$. Total cholesterol (TC), Triacylglycerol (TG), high-density lipoprotein cholesterol (HDL-C), and low-density lipoprotein cholesterol (LDL-C) kits were obtained from BioSino Bio-technology and Science Inc (Beijing, China). Superoxide dismutase (SOD) kits were purchased from Nanjing Jiancheng Bioengineering Institute (Wenzhou, China). Unless otherwise stated, all laboratory reagents were of analytical grade.

2.2. Animals and High-Fat Emulsions. Male Sprague-Dawley rats weighing $200 \pm 20 \mathrm{~g}$ were purchased from Guangzhou University of Chinese Medicine Laboratory Animal Center (Guangzhou, China). All animal protocols were approved by the institutional animal care and use committee of Guangdong Pharmaceutical University (Guangzhou, China). They were housed in an isolator caging system in an airconditioned animal room at $23 \pm 1^{\circ} \mathrm{C}$. Rats were allowed free access to food and water.

Briefly, the high-fat emulsions were prepared as follows. $10.0 \mathrm{~g}$ cholesterol and $1.0 \mathrm{~g}$ propylthiouracil powder were dissolved into $20.0 \mathrm{~g}$ lard oil at $80^{\circ} \mathrm{C}$ and stirred for $10 \mathrm{~min}$ to ensure complete dissolution as the oil phase. The primary emulsions were prepared by diluting $5 \mathrm{~mL}$ emulsifier (Tween-80) and $20 \mathrm{~mL}$ sodium deoxycholate solution $(2.0 \%)$ into the oil phase with a high-speed blender. Then, the distilled water was added to the primary emulsions to form the high-fat emulsions $(100 \mathrm{~mL})$ with stirring.

\subsection{Preparation and Characterization of Water-Soluble Chi-} tosan Nanoparticles. In this study, WSC-NPs were formed as a result of complex electrostatic interactions between the positively charged copolymers and negatively charged tripolyphosphate (TPP) under mild conditions. Briefly, WSC $(0.1 \% \mathrm{w} / \mathrm{v})$ and TPP $(0.1 \% \mathrm{w} / \mathrm{v})$ were dissolved in purified water. For preparation of WSC-NPs, the WSC solution $(500 \mathrm{~mL})$ was stirred $(800 \mathrm{rpm})$ at room temperature $\left(25^{\circ} \mathrm{C}\right)$. Then, $0.1 \%$ TPP solution $(100 \mathrm{~mL})$ was added to the system while stirring was continued to complete nanoparticles formation. The rate of adding TPP was $0.75 \mathrm{ml} / \mathrm{min}$. The nanosuspension was then spray dried using the Lab Spray
Dryer L-117 (Laiheng Scientific Co. Ltd, Beijing, China) with a standard nozzle $(0.5 \mathrm{~mm})$. The atomizing air flow rate was $10-15 \mathrm{~L} / \mathrm{min}$, and the flow rate was $600 \mathrm{ml} / \mathrm{h}$. The inlet temperature was controlled at $160^{\circ} \mathrm{C}$. The outlet temperature was determined by the inlet temperature and relative factors such as air and liquid feed flow rates and varied between $80-85^{\circ} \mathrm{C}$. The stability of WSC-NPs is affected by various environmental conditions during long-term storage. Studies were carried out to evaluate the stability of the WSC-NPs for 5 months at room temperature.

The FTIRs were taken with $\mathrm{KBr}$ pellets on Perkin-Elmer Spectrum one FTIR (Shimadzu, FT-IR 8700, Japan).The particle size and size distributions of the nanoparticles were performed by particle sizer (Zetasizer 3000 HAS, Malvern Instruments Ltd., Worcs, UK).The morphology of the nanoparticles was examined under scanning electron microscopy (SEM) using a Hitachi S3700N (Hitachi Ltd, Japan) microscope at $10 \mathrm{kV}$.

2.4. Experimental Procedure. The rats were fed ad libitum with a commercial diet for 5 days and were then assigned to 5 groups $(n=8)$ as follows: (a) normal diet fed rats (NF), (b) high-fat emulsions fed rats (HF), (c) highfat emulsions and $450 \mathrm{mg} / \mathrm{kg} / \mathrm{d}$ WSC fed rats (WSC), (d) high-fat emulsions and $450 \mathrm{mg} / \mathrm{kg} / \mathrm{d}$ WSC-NPs fed rats (HWSC-NP), and (e) high-fat emulsions and $225 \mathrm{mg} / \mathrm{kg} / \mathrm{d}$ WSC-NPs fed rats (L-WSC-NP). The NF group received an equivalent amount of distilled water; the HF group, received high-fat emulsions daily by oral intubation until the study ended. The other groups were administered the high-fat emulsions by oral intubation for 2 weeks to establish the hyperlipidemic condition, and then the WSC and WSC-NPs samples were administered orally to the WSC and WSC-NPs groups for 4 weeks. All groups were fed the corresponding diets in which the composition conformed to GB14924.3 (Guangdong Laboratory Animal Center, Guangzhou, China) as the basal diets during the whole experiment. Each rat was weighed once a week.

At the end of the experimental period, blood samples were withdrawn from the orbital venous plexus using a capillary tube under ether anesthesia after an overnight fast.

2.5. Serum Lipids and SOD. Blood was clotted at room temperature and centrifuged in a centrifuge at $3000 \mathrm{rpm}$ for 15 min. Serum was separated, and TC, TG, HDL-C, and LDL-C were measured with commercial assay kits using the Automated Biochemistry Analyzer AMS-18 (Beijing Option Science and Technology Development Co. Ltd, Beijing, China).

The serum SOD contents were analyzed with commercially available analytical kit by the SPECORD S600 UV-Vis Spectrophotometer (Analytic Jena AG, Germany).

2.6. Plasma Viscosity. Blood samples were taken from the ocular vein using a heparinized capillary tube and centrifuged at $3000 \mathrm{rpm}$ for $5 \mathrm{~min}$ in the Eppendorf Centrifuge 5810R (Eppendorf Co, Germany) to obtain the plasma. The plasma viscosity was measured by the Automatic Blood 


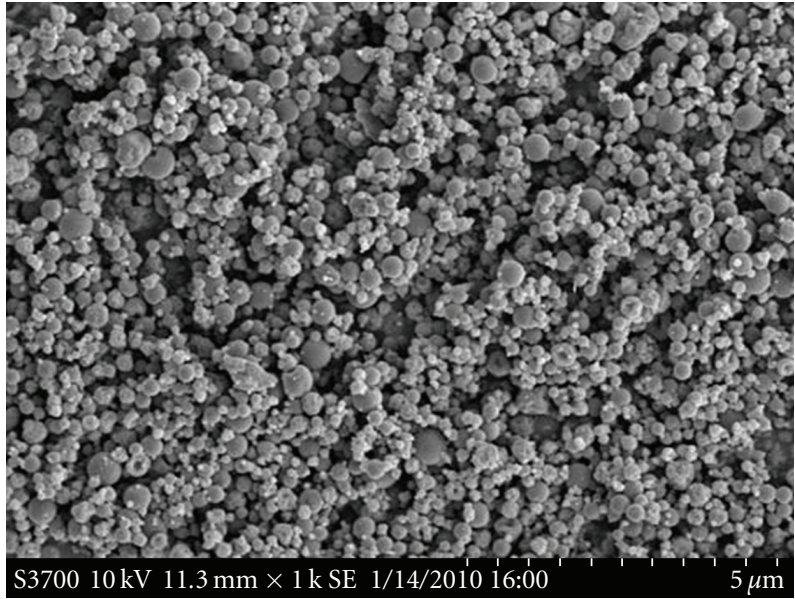

(a)

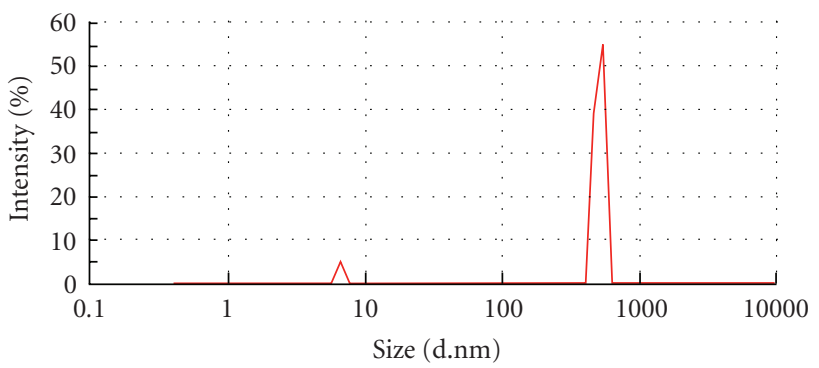

(b)

FIGURE 1: SEM (magnification of 1000x) microphotographs of WSC-NPs (a) and particle size distribution of WSC-NPs (b).

Rheometer LBY-N6B (Beijing Precil Instrument Co. Ltd, Beijng, China).

2.7. Statistical Analysis. All data were expressed as means \pm SE. Differences between the groups were determined by one-way analysis of variance, using a statistical analysis software program (SPSS for windows, version Rel, 16.0, Spss Inc, Chicago, IL); the Student-Newman-Keuls MultipleRange Test comparisons at $P$ value of $<.05$ were made to determine significant differences among means.

\section{Results and Discussions}

3.1. Characterizations and Stability of WSC-NPs. The microphotographs and particle size of the WSC-NPs are shown in Figure 1. All nanoparticles were found to be nearly spherical in shape, and the external surfaces appeared smooth (Figure 1(a)). The mean particles size of WSCNPs was $650 \mathrm{~nm}$ variing from 500 to $800 \mathrm{~nm}$ (Figure 1(b)). FTIR spectra of WSC-NPs and WSC matrix show that the tripolyphosphoric groups of TPP are linked with the ammonium group of WSC; the inter- and intramolecular actions are enhanced in WSC-NPs [15].

The stability studies show that there were no detectable changes in color, odor, taste, or $\mathrm{pH}$ and no visible microbial growth in the WSC-NPs.

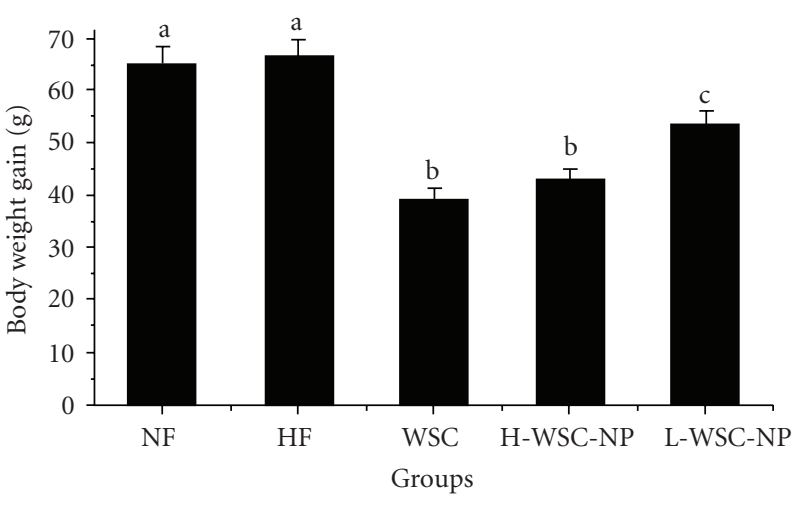

FIGURE 2: Effects on body weight gain in rats fed high-fat diets. Results are expressed as means $\pm \mathrm{SE}$ of eight rats. Values marked by the different letters are significantly different $(P<.05)$.

3.2. Effects on Body Weight in Rats Fed High-Fat Diets. A significantly lower weight gain was observed in all the treatment groups compared with the rats that consumed the normal diet and the high-fat diet (Figure 2), and H-WSCNP, WSC produced lower counts compared with L-WSC-NP. However, weight gains were not significant between the $\mathrm{H}$ WSC-NP and WSC groups.

Previously, WSC was shown to reduce body weight gain of rats that was caused by a high-fat diet [16]. In our study, the rats in the WSC group showed similar responses. Compared with the NF group, body weight gains of rats fed the WSC and H-WSC-NP were significantly lower than those fed the L-WSC-NP. This indicated that WSC could be used as weight-loss agent for healthy and obese humans, but further study is needed to clarify the antiobesity action and mechanisms of WSC-NPs.

3.3. Effects on Serum TC, TG, HDL-C, LDL-C, and SOD in Rats Fed High-Fat Diets. The hyperlipidemic model showing that the serum concentrations of TC, TG, HDL-C, and LDL-C increased, was established in the HF group after 2 weeks (Table 1). Compared with the HF group, the TC and TG levels in H-WSC-NP and L-WSC-NP groups were significantly decreased, showing that the effect of WSCNPs treatment was even more powerful than the treatment of WSC group. The serum LDL-C levels in the treatment groups were significantly decreased compared with the HF group, and no significant difference was observed among the treatment groups. There were no significant difference of HDL-C levels among all the groups (Table 1). The serum SOD was increased significantly in the H-WSC-NP and LWSC-NP groups compared with the NF and HF groups, but increased slightly in the WSC group (Table 2).

The low levels of HDL-C and the high levels of LDL-C indicated an imbalance between cholesterol transport from the liver to the extrahepatic tissues and back to the liver [17]. Moreover, dyslipidemia is characterized by increased fasting concentrations of total cholesterol (TC), triglycerides (TG), and LDL cholesterol (LDL-C), in conjunction with decreased concentrations of HDL cholesterol (HDL-C). WSC and 
TABLE 1: Effects on serum TC, TG, HDL-C, and LDL-C in rats fed high-fat diets.

\begin{tabular}{lcccc}
\hline Group & TC $(\mathrm{mmol} / \mathrm{L})$ & TG $(\mathrm{mmol} / \mathrm{L})$ & HDL $(\mathrm{mmol} / \mathrm{L})$ & LDL $(\mathrm{mmol} / \mathrm{L})$ \\
\hline NF & $1.59 \pm 0.21^{\mathrm{a}}$ & $0.99 \pm 0.20^{\mathrm{d}}$ & $0.70 \pm 0.07^{\mathrm{f}}$ & $1.03 \pm 0.18^{\mathrm{h}}$ \\
HF & $2.45 \pm 0.28^{\mathrm{b}}$ & $1.46 \pm 0.23^{\mathrm{e}}$ & $1.19 \pm 0.14^{\mathrm{g}}$ & $1.33 \pm 0.19^{\mathrm{i}}$ \\
WSC & $1.52 \pm 0.78^{\mathrm{a}}$ & $1.33 \pm 0.37^{\mathrm{e}}$ & $0.62 \pm 0.17^{\mathrm{f}}$ & $0.78 \pm 0.25^{\mathrm{j}}$ \\
H-WSC-NP & $0.46 \pm 0.69^{\mathrm{c}}$ & $1.02 \pm 0.33^{\mathrm{d}}$ & $0.67 \pm 0.26^{\mathrm{f}}$ & $0.67 \pm 0.32^{\mathrm{j}}$ \\
L-WSC-NP & $0.33 \pm 0.24^{\mathrm{c}}$ & $0.82 \pm 0.50^{\mathrm{d}}$ & $0.91 \pm 0.33^{\mathrm{fg}}$ & $0.92 \pm 0.26^{\mathrm{j}}$ \\
\hline
\end{tabular}

Values are expressed as means $\pm \mathrm{SE}(n=8)$. Values marked by different letters within the same row are significantly different $(P<.05)$.

TABLE 2: Effects on serum SOD in rats fed high-fat diets.

\begin{tabular}{lc}
\hline Group & Serum SOD $(\mathrm{U} / \mathrm{mL})$ \\
\hline NF & $83.43 \pm 9.78^{\mathrm{a}}$ \\
HF & $87.18 \pm 14.01^{\mathrm{a}}$ \\
WSC & $92.35 \pm 6.32^{\mathrm{b}}$ \\
H-WSC-NP & $107.23 \pm 16.25^{\mathrm{c}}$ \\
L-WSC-NP & $98.08 \pm 12.68^{\mathrm{b}}$ \\
\hline
\end{tabular}

Values are expressed as means $\pm \mathrm{SE}(n=8)$. Values marked by the different letters within the same row are significantly different $(P<.05)$.

WSC-NPs significantly lowered TC, TG, and LDL-C levels in the plasma, which was consistent with previous reports [18]. Plasma concentration of HDL is inversely correlated with the risk of coronary heart disease, but low HDL-C poses no risk in the absence of elevated LDL cholesterol or TC. The levels of plasma HDL-C should be increased in the WSC-NP group; however, plasma concentration of HDL is influenced by several factors, including gender, race, and diet. Furthermore, WSC-NPs exhibited better cholesterolbinding capacity than WSC. This is consistent with the previous report that chitosan with finer particle size could effectively lower the plasma and liver lipid level in rats [19].

Excessive superoxide radicals may induce lots of senile diseases such as atherosclerosis. Any elevation in the level of the SOD was accompanied by a decrease in superoxide radicals [20, 21]. In the experiment, the serum SOD was elevated significantly by feeding the H-WSCNP and L-WSC-NP compared with NF and HF groups, and WSC could not increase the serum SOD significantly. Therefore, it seems likely that WSC-NPs may improve the hypercholesterolemia induced by the high-fat diet through reducing serum $\mathrm{TC}, \mathrm{TG}$, and $\mathrm{LDL}-\mathrm{C}$, and elevating the SOD activity.

3.4. Effects on Plasma Viscosity in Rats Fed High-Fat Diets. As shown in Table 3, by experimental design, the average plasma viscosity of rats in the HF group increases significantly compared with the average level for rats in the NF group. In the three treatment groups, plasma viscosity showed a decreasing trend. When compared with the HF group, significant differences were seen for the H-WSC-NP and LWSC-NP groups.
TABLE 3: Effects on plasma Viscosity in rats fed high-fat diets.

\begin{tabular}{lc}
\hline Group & Plasma viscosity $(\mathrm{mPa} . \mathrm{S})$ \\
\hline NF & $1.47 \pm 0.25^{\mathrm{a}}$ \\
HF & $1.84 \pm 0.40^{\mathrm{b}}$ \\
WSC & $1.68 \pm 0.18^{\mathrm{b}}$ \\
H-WSC-NP & $0.86 \pm 0.18^{\mathrm{c}}$ \\
L-WSC-NP & $1.13 \pm 0.50^{\mathrm{c}}$
\end{tabular}

Values are expressed as means $\pm \mathrm{SE}(n=8)$. Values marked by different letters within the same row are significantly different $(P<.05)$.

Plasma viscosity played an important role in the perfusion of the microvasculature and was a major determinant of endothelial shear stress [22]. Plasma viscosity was used as a marker for different diseases in humans such as coronary artery disease atherosclerosis [23]. The rats' plasma viscosity was increased significantly by feeding the high-fat emulsions, and the WSC-NPs and WSC reduced this increase effectively. Although the plasma viscosity tended to decrease in the WSC group, the differences were not statistically significant compared with HF group. The results suggested that the mechanisms of WSC to improve TC, TG, LDL, and SOD may different with plasma viscosity. Furthermore, the WSC-NPs exhibited a better effect than the WSC, showing that WSC-NPs may serve as a useful agent for preventing hypercholesterolemia.

\section{Conclusions}

In conclusion, the data generated by this study demonstrated that WSC-NPs not only lower serum lipids levels and plasma viscosity but also increased serum SOD activities. Moreover, the hypercholesterolemia is affected by WSC-NPs even more than the WSC. Hence, the data obtained from this study could facilitate the further development of dietary intervention to hypercholesterolemia. Further studies are needed to clarify the mechanisms of the WSC-NPs to inhibit hypercholesterolemia induced by feeding a high-fat diet in male Sprague-Dawley rats.

To date, all rats appear healthy and remain active after oral administration of the WSC and WSC-NPs. Therefore, WSC and WSC-NPs are safe dietary fibers to inhibit hypercholesterolemia. 


\section{Abbreviations}

$\begin{array}{ll}\text { WSC: } & \text { Water-soluble chitosan } \\ \text { WSC-NP: } & \text { Water-soluble chitosan nanoparticle } \\ \text { SOD: } & \text { Superoxide dismutase } \\ \text { TC: } & \text { Total cholesterol } \\ \text { TG: } & \text { Triglycerides } \\ \text { HDL-C: } & \text { High-density lipoprotein cholesterol } \\ \text { LDL-C: } & \text { Low-density lipoprotein cholesterol } \\ \text { TPP: } & \text { Tripolyphosphate. } \\ \text { SEM: } & \text { Scanning electron microscopy } \\ \text { FTIR: } & \text { Fourier transform infrared spectroscopy } \\ \text { NF: } & \text { Normal diet fed rats } \\ \text { HF: } & \text { High-fat emulsions fed rats } \\ \text { H-WSC-NP: } & \text { High-fat emulsions and } 450 \mathrm{mg} / \mathrm{kg} / \mathrm{d} \\ & \text { WSC-NPs fed rats } \\ \text { L-WSC-NP: } & \text { High-fat emulsions and } 225 \mathrm{mg} / \mathrm{kg} / \mathrm{d} \\ & \text { WSC-NPs fed rats. }\end{array}$

\section{Acknowledgments}

This paper was financially supported by Science and Technology Planning Project of Guangdong Province, China (no. 2009B020313006) and Science and Technology Planning Project of Zhongshan, China (no. 2009H017), and Foundation for University Key Teacher of Guangdong Pharmaceutical University, China.

\section{References}

[1] K. A. Varady and P. J. H. Jones, "Combination diet and exercise interventions for the treatment of dyslipidemia: an effective preliminary strategy to lower cholesterol levels?" Journal of Nutrition, vol. 135, no. 8, pp. 1829-1835, 2005.

[2] Y. Luo, G. Chen, B. Li et al., "Dietary intervention with AHP, a functional formula diet, improves both serum and hepatic lipids profile in dyslipidemia mice," Journal of Food Science, vol. 74, no. 6, pp. H189-H195, 2009.

[3] E. S. Ostanina, V. P. Varlamov, and G. I. Iakovlev, "Inhibition of lipase activity by low-molecular-weight chitosan," Prikladnaia biokhimiia i mikrobiologiia, vol. 44, no. 1, pp. 38-43, 2008.

[4] C. L. Bartels and S. J. Miller, "Dietary supplements marketed for weight loss," Nutrition in Clinical Practice, vol. 18, no. 2, pp. 156-169, 2003.

[5] M. R. Rekha and C. P. Sharma, "Synthesis and evaluation of lauryl succinyl chitosan particles towards oral insulin delivery and absorption," Journal of Controlled Release, vol. 135, no. 2, pp. 144-151, 2009.

[6] N. Bhattarai, H. R. Ramay, J. Gunn, F. A. Matsen, and M. Zhang, "PEG-grafted chitosan as an injectable thermosensitive hydrogel for sustained protein release," Journal of Controlled Release, vol. 103, no. 3, pp. 609-624, 2005.

[7] G. Y. Park, S. Mun, Y. Park et al., "Influence of encapsulation of emulsified lipids with chitosan on their in vivo digestibility," Food Chemistry, vol. 104, no. 2, pp. 761-767, 2007.

[8] J. Zhang, J. Liu, L. Li, and W. Xia, "Dietary chitosan improves hypercholesterolemia in rats fed high-fat diets," Nutrition Research, vol. 28, no. 6, pp. 383-390, 2008.

[9] S. F. Ausar, M. Morcillo, A. E. León et al., "Improvement of HDL- and LDL-cholesterol levels in diabetic subjects by feeding bread containing chitosan," Journal of Medicinal Food, vol. 6, no. 4, pp. 397-399, 2003.
[10] T. Fukamizo and R. Brzezinski, "Chitosanase from Streptomyces sp. strain N174: a comparative review of its structure and function," Biochemistry and Cell Biology, vol. 75, no. 6, pp. 687-696, 1997.

[11] H. Yin, Y. Du, and J. Zhang, "Low molecular weight and oligomeric chitosans and their bioactivities," Current Topics in Medicinal Chemistry, vol. 9, no. 16, pp. 1546-1559, 2009.

[12] Y. Maeda and Y. Kimura, "Antitumor effects of various lowmolecular-weight chitosans are due to increased natural killer activity of intestinal intraepithelial lymphocytes in sarcoma 180-bearing mice," Journal of Nutrition, vol. 134, no. 4, pp. 945-950, 2004.

[13] M. Sumiyoshi and Y. Kimura, "Low molecular weight chitosan inhibits obesity induced by feeding a high-fat diet long-term in mice," Journal of Pharmacy and Pharmacology, vol. 58, no. 2, pp. 201-207, 2006.

[14] H. Hoyer, W. Schlocker, M. Greindl, T. Ostermann, and A. Bernkop-Schnurch, "Preparation and evaluation of thiomer nanoparticles via high pressure homogenization," Journal of Microencapsulation, vol. 27, no. 6, pp. 487-495, 2010.

[15] H. L. Zhang, S. H. Wu, Y. Tao, L. Q. Zang, and Z. Q. Su, "Preparation and characterization of water-soluble chitosan nanoparticles as protein delivery system," Journal of Nanomaterials, vol. 2010, Article ID 898910, 5 pages, 2010.

[16] H. G. Choi, J. K. Kim, D. H. Kwak et al., "Effects of high molecular weight water-soluble chitosan on in vitro fertilization and ovulation in mice fed a high-fat diet," Archives of Pharmacal Research, vol. 25, no. 2, pp. 178-183, 2002.

[17] S. Hossain, A. Rahman, Y. Kabir, A. A. Shams, F. Afros, and M. Hashimoto, "Effects of shrimp (M0acrobracium rosenbergii)derived chitosan on plasma lipid profile and liver lipid peroxide levels in normo- and hypercholesterolaemic rats," Clinical and Experimental Pharmacology and Physiology, vol. 34, no. 3, pp. 170-176, 2007.

[18] D. J. Ormrod, C. C. Holmes, and T. E. Miller, "Dietary chitosan inhibits hypercholesterolaemia and atherogenesis in the apolipoprotein E-deficient mouse model of atherosclerosis," Atherosclerosis, vol. 138, no. 2, pp. 329-334, 1998.

[19] M. Sugano and Y. Fujisaki, "Effect of the type of diet on the distribution of 3-hydroxy-3-methylglutaryl coenzyme A (HMG-CoA) reductase in rat small intestine," Experientia, vol. 36, no. 12, pp. 1399-1400, 1980.

[20] F. Kimura, G. Hasegawa, H. Obayashi et al., "Serum extracellular superoxide dismutase in patients with type 2 diabetes: relationship to the development of micro- and macrovascular complications," Diabetes Care, vol. 26, no. 4, pp. 1246-1250, 2003.

[21] R. A. DiSilvestro, "Influence of copper intake and inflammation on rat serum superoxide dismutase activity levels," Journal of Nutrition, vol. 118, no. 4, pp. 474-479, 1988.

[22] U. Windberger, A. Bartholovitsch, R. Plasenzetti, K. J. Korak, and G. Heinze, "Whole blood viscosity, plasma viscosity and erythrocyte aggregation in nine mammalian species: reference values and comparison of data," Experimental Physiology, vol. 88, no. 3, pp. 431-440, 2003.

[23] F. G. R. Fowkes, J. P. Pell, P. T. Donnan et al., "Sex differences in susceptibility to etiologic factors for peripheral atherosclerosis: importance of plasma fibrinogen and blood viscosity," Arteriosclerosis and Thrombosis, vol. 14, no. 6, pp. 862-868, 1994. 

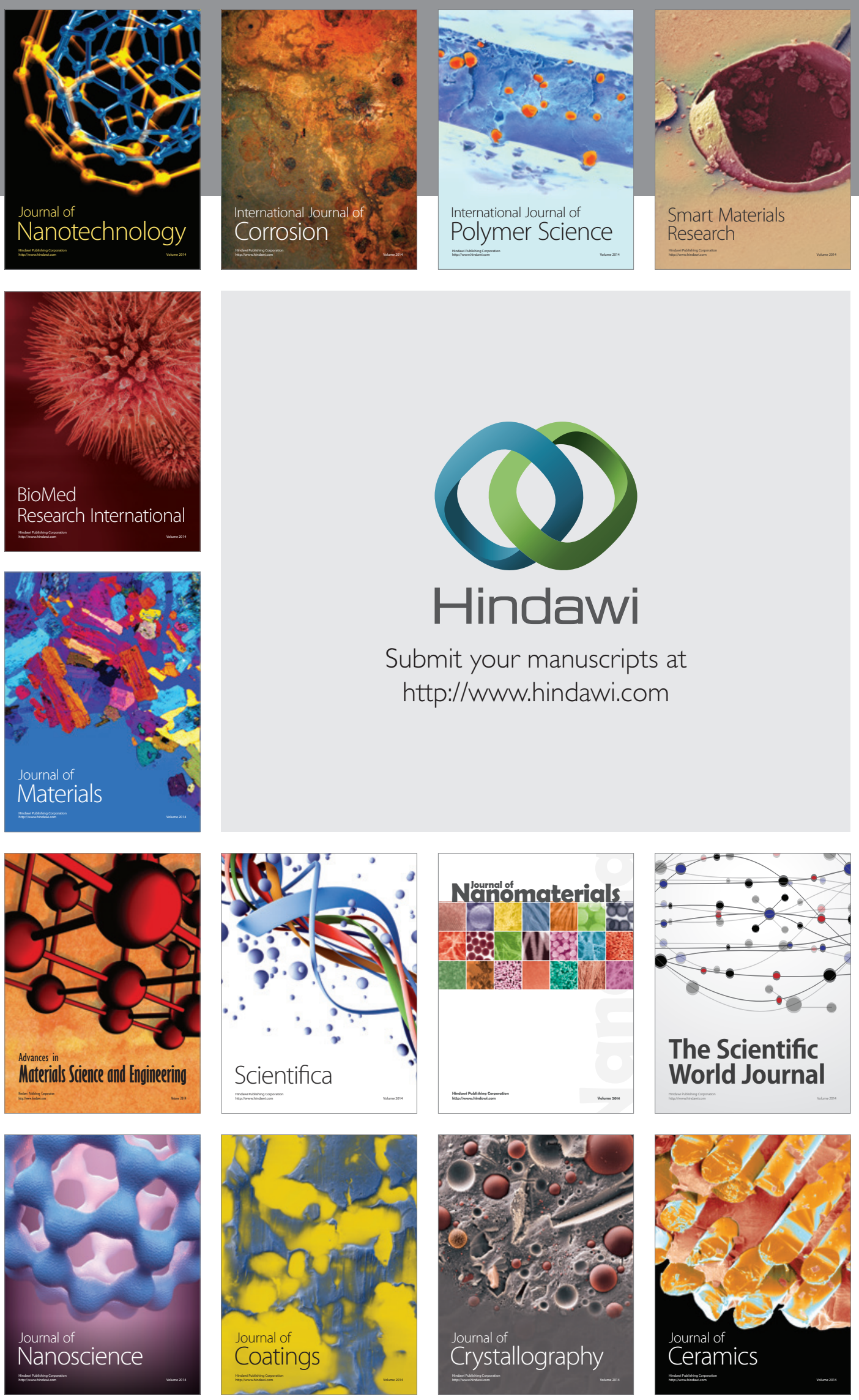

The Scientific World Journal

Submit your manuscripts at

http://www.hindawi.com

\section{World Journal}

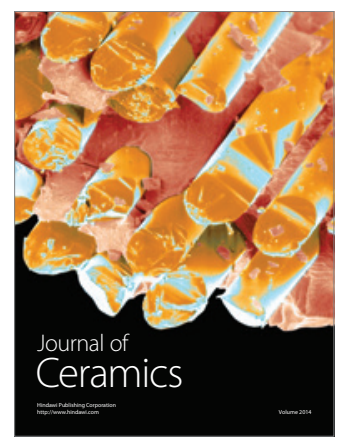

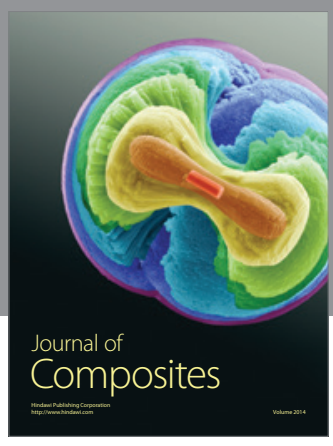
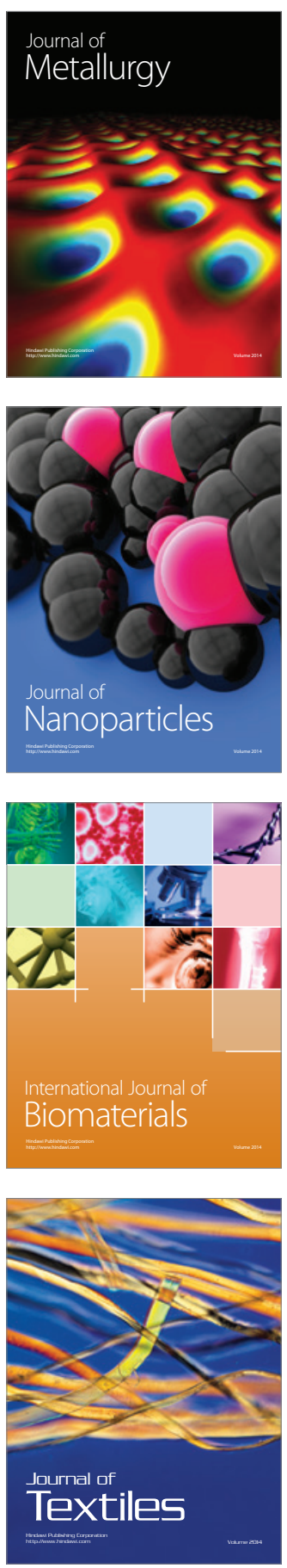